\title{
Study on Association between Iron Deficiency Anemia and Febrile Seizures in Childhood
}

\author{
Dr. Sathiyasuresh. R M.D ${ }^{1}$, Dr. Suresh Kumar. S M. D \\ ${ }^{1}$ Assistant Professor Govt Thiruvarur Medical College, Thiruvarur \\ ${ }^{2}$ Senior Assistant Professor, Chengalpattu Medical College, Chengalpattu
}

\begin{abstract}
Introduction: Febrile seizures are the most common form of childhood seizures, occurring in 2 to $5 \%$ of children. The peak incidence is at the age of approximately 18 months, with recurrent episodes occurring in one third of patients. Iron deficiency is the most common nutritional disorder affecting at least one third of world's especially between 6 and 24 months, this study was taken up to determine the association between febrile seizures and Iron deficiency as defined by the hematological parameters, which is a very important investigation in a resource limited country like India. AIMS: Prospective study to determine the association between iron deficiency anemia and febrile seizures in the age group 6 to 60 months. Methods and Materials: study design Case control study. Study period 7 months period from March 2014 to September 2014 Children of age group 6 months to 60 months presenting with febrile seizures to the pediatric emergency department and wards of the hospital were included Results: $80 \%$ of the study population within seizure group was between 6 months and 36 months. There was no significant gender predisposition for febrile seizures, and both genders were affected more or less in equal proportion.Family history of febrile seizures was a significant non modifiable risk factor in the seizure group. 21.4\% of seizure group had a family history of febrile seizures in first degree relatives. There was a significant difference in the value of $\mathrm{Hb}, \mathrm{MCH}$, serum iron, TIBC and RDW among the seizure group as compared to control group. There was a mean difference of 5\% of MCV between the seizure group and controls, which was not statistically significant in the study. Conclusion: Hematological parameters have been demonstrated to be sensitive and specific in children, and stable in the presence of febrile illness. Complete Blood count, being a simple and feasible investigation, can be used to screen individuals with iron deficiency in simple febrile seizures, for making a decision to start iron supplementation.
\end{abstract}

Keywords: Febrile seizures, Iron deficiency anemia, Fever, Hemoglobin, Hematological parameters

\section{Introduction}

Febrile seizures are the most common form of childhood seizures. [1] These episodes are agonizing to the parents and child and therefore can cause psychological trauma to both. Although frightening to the caregivers, they are benign, and the risk of subsequent epilepsy population. Febrile convulsions have been studied extensively over the past two decades, and a large body of literature now exists to help practitioners assess the risks associated with such presentations. Currently identified risk factors for febrile seizures include having a first or second degree relative with a history of febrile seizure, maternal smoking in pregnancy, low birth weight, neonatal stay of $>30$ days, attendance at day care, increased number of febrile illnesses per year, Fever > 39.4, and particular infectious illnesses. [2] Genetic factors contribute significantly to the etiology. Most studies have supported a polygenic or multi factorial model; with an estimated heritability of $75 \%$ [3-5] despite these studies the risk factors remain largely unknown. Iron deficiency is the most common nutritional disorder affecting at least one third of world's especially between 6 and 24 months. [6-9] Iron deficiency is defined as a condition in which there are no mobilizable iron stores and in which signs of a compromised supply of iron to tissues, including the erythron, are noted. [10] The more severe stages of iron deficiency are associated with anemia and though anemia is the most common manifestation of Iron deficiency, other effects of Iron deficiency of various tissues and organs are still under recognized and needs more researches in the field. Impaired brain developments along with cognitive, behavioral and psychomotor manifestations are still under recognized to a larger extent. It has been shown by various studies that some of these impairments on the brain during the first two years, i.e. the growth spurt period of brain may be irreversible. Among numerous biological effects of iron, there is considerable evidence that iron is also important for the neurological functioning, which include neurotransmitter metabolism, myelin formation, and brain energy metabolism. Iron- deficient animals show alterations both in neurotransmitters and behavior that do not usually respond to iron replenishment. As iron is important for the growth of neurotransmitters and various enzymes, iron deficiency may lower the seizure threshold. In a resource country like India, it is not always feasible to get the biochemical parameters of Iron stores in the body, and hematological parameters available in a complete blood count is not interpreted in a systematic way to screen Iron deficiency anemia. There is no single standard test to assess iron deficiency without anemia.[10] so as a result of this many Microcytic hypochromic anemia's of which Iron deficiency anemia being the most common cause, go unnoticed and untreated. So this study was taken up to determine the association between febrile seizures and Iron deficiency as defined by the hematological parameters, which is a very important investigation in a resource limited country like India. And if such an association exists, iron deficiency can be addressed at an earlier stage which may be helpful for prevention of simple febrile seizures

\section{Aim}

Prospective study to determine the association between iron deficiency anemia and febrile seizures in the age group 6 to 60 months.

\section{Volume 6 Issue 1, January 2017




\section{International Journal of Science and Research (IJSR)}

ISSN (Online): 2319-7064

Index Copernicus Value (2015): 78.96 | Impact Factor (2015): 6.391

\section{Methods and Materials}

\section{Study Desing}

Case control study study period: 7 months period from March 2014 to September 2014 subjects: This was a prospective case control study done in a tertiary care hospital (chengalpattu Medical Hospital) between the periods March 2014 to September 2014. Ethical clearance was obtained for the study from the Ethical Committee of the Hospital.

\section{Inclusion Criteria}

Children of age group 6 months to 60 months presenting with febrile seizures to the pediatrics emergency department and wards of the hospital during the study period. Diagnostic criteria for simple febrile seizures (based on AAP Clinical Practice Guidelines: 2008) included seizures associated with fever and the seizures were generalized, short duration (less than 15 minutes), no recurrence of seizures within 24 hours, child is otherwise neurologically healthy and without any neurological abnormality before and after the episode of seizures, with age group between 6 months to 5 years. (9)

\section{Exclusion Criteria}

Children with age less than 6 months and more than 60months.children with Development delay, Central neurons system infections, Past history of non febrile seizure, Chronic multisystem diseases, Diagnosed cases of other hematological problems like hemolytic anemia's, bleeding (or) coagulation disorders, hematological malignancies (or) Children on Haematinics were excluded After informed consent, detailed history was elicited and physical examination was done Hospital records were also examined for relevant data. Blood Investigations done to diagnose Iron deficiency included hemoglobin $(\mathrm{Hb})$ in $\mathrm{gm} / \mathrm{dl}$ estimation comparisons for the categorical variables were analyzed using chi square test and within group comparison of quantity variables were analyzed using independent test. The $\mathrm{P}$ value of less than 0.05 was considered as statistically significant. The statistical analysis was carried out using statistical software SPSS 19.

\section{Results}

The mean age of seizure group and controls were $20.73 \pm$ 12.43 and $23.10 \pm 14.17$ months respectively. Also both the groups had almost similar proportions of males $(51.4 \%$ and $48.6 \%)$ and Females $(61.4 \%)$ and $(38.6 \%)$ Also the mean temperature in the seizure group was found to be $100.36 \pm$ 0.55 . $80 \%$ of the Study populations in the seizure group were between 6 months and 36 months of age, with the mean of $20.73 \pm 12.43$ months and there was no significant difference in gender predilection. In this study $42.9 \%$ of seizure group had peripheral smear shows microcytic hypochromic, whereas only $10 \%$ of controls were peripheral smear shows microcytic hypochornic anemia. This was found to be statistically significant $(\mathrm{P}-0.000)$. respiratory tract infections, viral fever and acute diappoheal disease were the most frequent underlying illnesses for fever in and mean corpuscular volume (MCV) in $\mathrm{Fl}$, Mean corpuscular Hemoglobin in $(\mathrm{MCH})$ in $\mathrm{Pg}$ and red cell distribution width (RDW) in percentage, Reticulocyte count in percentage, serum Iron in $(\mathrm{Mg} / \mathrm{dl})$ (Microgram/ deciliter, serum total iron binding capacity in Microgram/ deciliter and peripheral smear study. The investigations was carried out using an automated Hematology Analyser. Iron deficiency was diagnosed by hematologic investigations of hemoglobin value $<11 \mathrm{gm} / \mathrm{dl}, \mathrm{MCV}<70 \mathrm{Fl}, \mathrm{MCH}<27 \mathrm{Pg}$ and RDW > 15\%, serum Iron < $60 \mathrm{Mg} / \mathrm{dl}$, TIBC $>400$ $\mathrm{Mg} / \mathrm{dl}$, Reticulocyte count Normal (2-2.5\%). Peripheral smear-shows microcytic hypochromic. Other variables studied included age of the child, sex, family history of febrile seizure in first degree relatives, neonatal hospital admissions (NICU or special care nursery admissions), permaturity ( $<37$ weeks gestational), nature of underlying illness and immunization status of child.The controls were selected from the same setting and included febrile children of age group 6 months to 60 months who presented with short duration fever ( $<3$ days) but without seizures to the outpatient department or wards. Cases and controls were selected in 1:1 ratio. No matching was done.

\section{Sample Size}

Based on the results form available publications in India, and with $90 \%$ power and $99 \%$ confidence minimum sample size in each group come to 70 each. Data were entered in MS Excel, cleaned and checked. The statistical analysis was carried out using statistical software SPSS19.

\section{Statistics Analysis}

The categorical variables were expressed as Frequency and percentage. The quantity variables were expressed as mean \pm standard deviation. Descriptive statistics were used to evaluate baseline characteristics. The group cases, whereas Respiratory tract infections and viral fever were more among the control group. Other few cases have diagnosed urinary tract infection. Also acute respiratory tract infecctions were the most frequent causes for fever in control. In this study $21.4 \%$ of seizure group had a family history of febrile seizures in first degree relatives, whereas only $2.9 \%$ of controls were having a positive family history (P-0.000) Family history of febrile seizures, which most likely presents a genetic susceptibility to seizures with fever, may be a significant non modifiable risk factor. This study also demonstrates that Iron deficiency as defined by hematological parameters i.e., $\mathrm{Hb}, \mathrm{MCV}, \mathrm{MCH}, \mathrm{RDW}$, serum Iron, serum ITBC, Reticulocyte count and peripheral smear was significantly associated with seizure group (43\%) when compared with controls $(10 \%)$ ( $\mathrm{P}$ value 0.0001$)$ The results of this study demonstrated significantly lower levels of HB, MCH, Serum Iron, Serum TIBC and higher values of RDW among the cases with febrile seizures that in the controls $(\mathrm{P}<0.05)$. This was found to be statistically significant. MCV was low in cases as compared with controls, but a mean difference of $5 \%$ of MCV between the seizure group and controls, but differences failed to attain statistical significance 


\section{International Journal of Science and Research (IJSR) \\ ISSN (Online): 2319-7064}

Index Copernicus Value (2015): 78.96 | Impact Factor (2015): 6.391

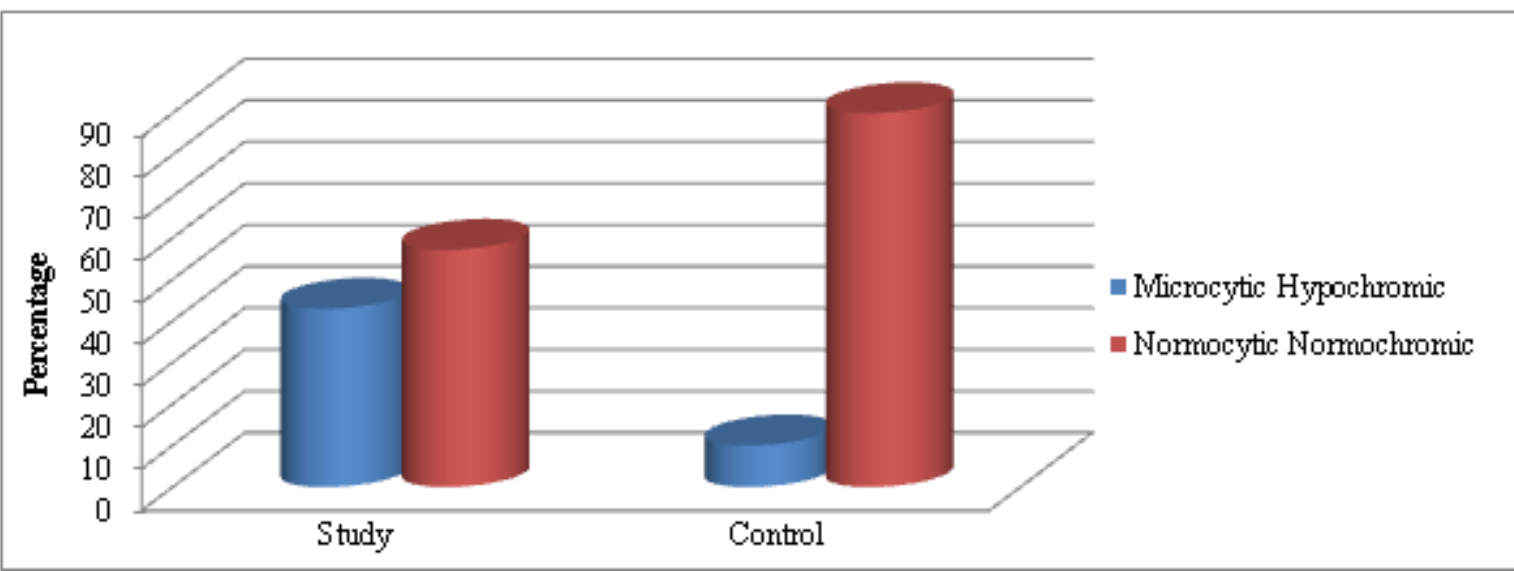

Figure 1: Peripheral Smear Distribution
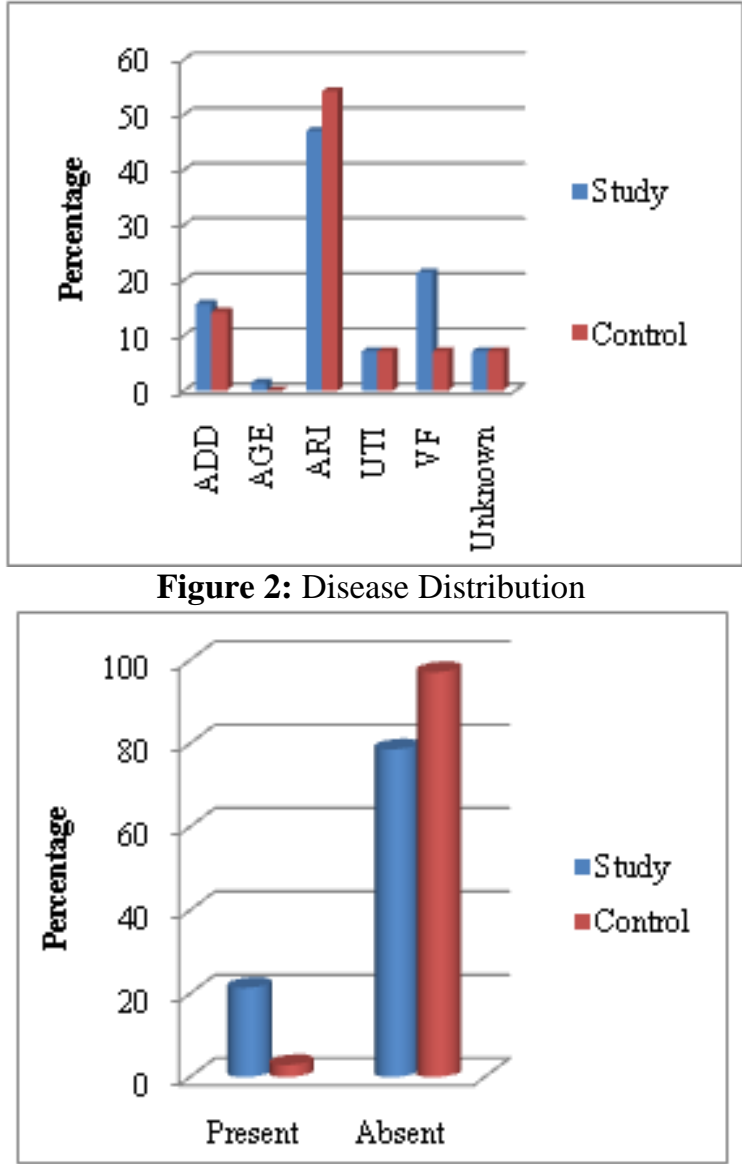

Figure 3: Family History of Febrile Seizure

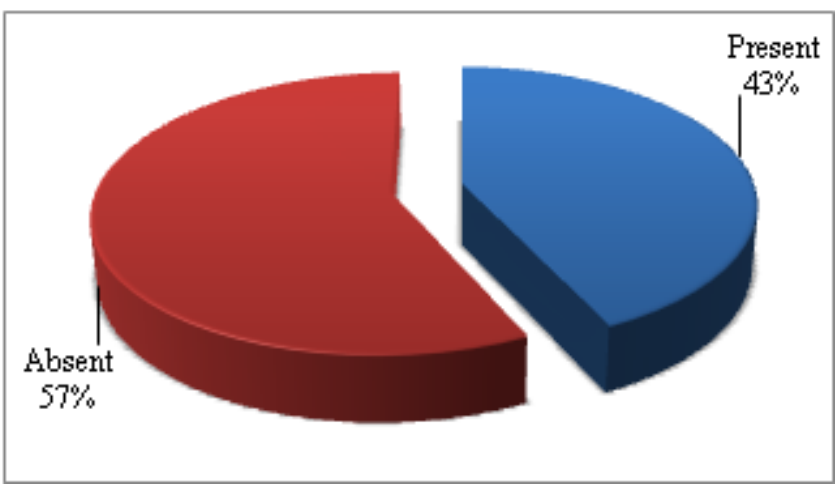

Figure 4: Iron Deficiency Anemia in Study Group

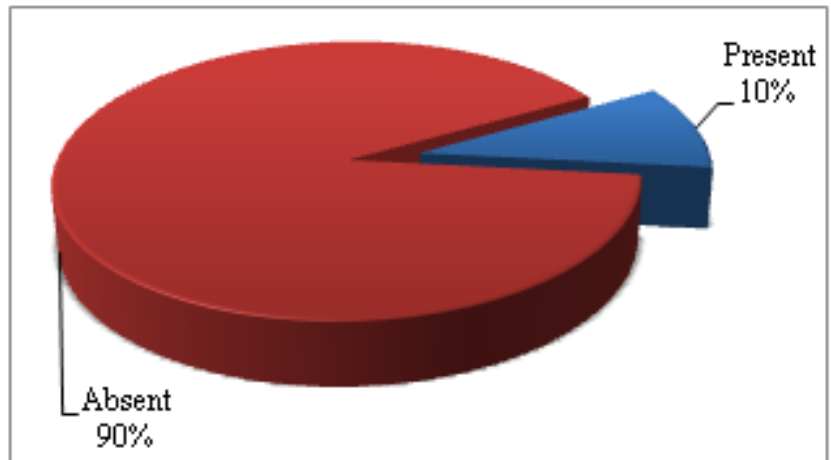

Figure 5: Iron Deficiency Anemia In Control Group

Table 1: Comparison of Cases and Controls With Respect To Variables.

\begin{tabular}{|c|c|c|c|}
\hline & Study & Control & P Value \\
\hline Age & $20.73 \pm 12.43$ & $23.10 \pm 14.17$ & 0.294 \\
\hline HB & $10.62 \pm 1.39$ & $11.43 \pm 0.81$ & $<0.05$ \\
\hline MCV & $76.06 \pm 5.42$ & $78.63 \pm 3.24$ & 0.001 \\
\hline MCH & $25.01 \pm 3.13$ & $26.56 \pm 1.23$ & $<0.05$ \\
\hline RDW & $14.77 \pm 1.38$ & $13.90 \pm 0.89$ & $<0.05$ \\
\hline Reticulocyte Count & $2.16 \pm 0.24$ & $2.19 \pm 0.12$ & 0.504 \\
\hline Serum Iron & $50.89 \pm 25.99$ & $78.92 \pm 19.33$ & $<0.05$ \\
\hline TIBC & $508.15 \pm 145.18$ & $412.24 \pm 76.08$ & $<0.05$ \\
\hline Temperature $(\mathrm{F})$ & $100.36 \pm 0.55$ & $99.91 \pm 0.29$ & 0.000 \\
\hline
\end{tabular}

\section{Discussion}

In this study, $80 \%$ of the study population within seizure group was between 6 months and 36 months. There was no significant gender predisposition for febrile seizures, and both genders were affected more or less in equal proportion. Pisacane et al. [11] in a case-control study with 146 cases and 146 controls, with age of 6-24 months, reported a significantly higher rate of iron deficiency anemia among children with first episode of febrile convulsions than in controls. Iron status was measured by $\mathrm{HB}, \mathrm{MCV}$ and serum Iron. Dawn et al. [12] in a retrospective case control study of 361 cases and 390 controls in the age group 6 to 36 months found that children with febrile seizure were almost twice as likely to be iron deficient as those with febrile illnesses alone. Iron status was measured by $\mathrm{HB}, \mathrm{MCV}$ and RDW. Daoud et al. [13] in a case-control study with 75 cases and 75 controls in the age group of 3-72 months, demonstrated that only low PF level is associated with and may play a role in first episode of febrile convulsions, with lack of

\section{Volume 6 Issue 1, January 2017 www.ijsr.net}




\section{International Journal of Science and Research (IJSR) \\ ISSN (Online): 2319-7064}

Index Copernicus Value (2015): 78.96 | Impact Factor (2015): 6.391

significant differences in $\mathrm{HB}, \mathrm{MCV}$ and $\mathrm{MCH}$. Iron status was measured by $\mathrm{HB}, \mathrm{MCV}, \mathrm{MCH}$ and serum Ferritin. At the same time Kobrinsky et al. [14] in a case-control study with 25 cases and 26 controls in the age group of 6-36 months concluded that children with febrile seizures were less likely to be iron deficient and iron deficiency may protect against the development of febrile convulsions. Similarly, Elham Bidabadi a, Mehryar Mashouf, [15] in a case-control study with 200 cases and controls suggested that iron deficiency anemia was less frequent among the cases with febrile convulsion, as compared to the controls, and there is not a protective effect of iron deficiency against febrile convulsions. Bidabadi et $\mathrm{al},[15]$ in a case control study with 200 cases and 200 controls suggested that iron deficiency anemia was less frequent among the cases with febrile convulsions in age group of 6months to 5 years. In this study,the amount of RBC, serum iron and plasma ferritin were high and TIBC was low among the cases with first febrile convulsions than in the controls. The amount of $\mathrm{Hb}, \mathrm{HCT}, \mathrm{MCV}, \mathrm{MCH}$, and MCHC were high among the cases than controls, but differences were no statistically significant. Iron deficiency anemia was less frequent among the cases with febrile convulsions, as compared to the controls, and its difference was not statistically significant. Derakshanfar H et al[16] in this case control study done in 500 children with febrile seizure (cases) and 500 children without febrile seizures( controls) in the age group of 6-60 months, suggested that the risk of febrile seizures in anemic children is less common than non- anemic ones. The amount of hemoglobin, hematocrit, $\mathrm{MCV}, \mathrm{MCH}, \mathrm{MCHC}, \mathrm{Rbc}$ count, serum iron and plasma ferritin were significantly higher and TIBC was significantly lower among the cases with febrile convulsions than the controls. The incidence of iron deficiency anemia was significantly higher in controls compared with the cases ( $p$ less than 0.016). The mean of temperature peak on admission was significantly higher in the febrile convulsions cases than in controls. Hartfield DS et al [17] conducted a retrospective case control study with 361 cases with febrile seizures and 390 controls presented with febrile illness without seizures in the age group of 6 to 36 months. This study used MCV, RDW and hemoglobin to determine the iron status. The results demonstrated that $9 \%$ of cases had iron deficiency anemia compared to $5 \%$ of controls and $6 \%$ had iron deficiency compared to $4 \%$ of controls. The conditional logistic regression odds ratio for iron deficiency in patients with febrile seizures was 1.84 . The study conclusion is that febrile seizures were almost as twice common in iron deficiency anemia. The results also suggested that screening for iron deficiency anemia should be considered in children presenting with febrile seizures. In this study the majority of febrile convulsions occur between 6 months and 36 months of age, with the mean of $20.73 \pm$ 12.43 months and there was no significant difference in sex predilection. Also, family history of febrile seizure, which most likely represents a genetic susceptibility to seizures with fever, were higher among seizure group ( $\mathrm{P}<$ 0.000). The results of our study demonstrated significantly lower levels of $\mathrm{Hb}, \mathrm{MCH}$, serum Iron, TIBC and higher values of RDW among the seizure group when compared with controls $(\mathrm{P}<0.05)$. The amount of MCV, Hct, MCHC and $\mathrm{RBC}$ count were also lower in the seizure group as compared with controls, but differences failed to attain statistical significance. There was a mean difference of $5 \%$ of MCV between the seizure group and controls, which was not statistically significant in the study. In our study $42.9 \%$ of seizure group had peripheral smear shows microcytic hypochromic, whereas only $10 \%$ of controls were peripheral smear shows microcytic hypochromic anemia. This was found to be statistically significant $(P-0.000)$ The study also demonstrates that Iron deficiency as defined by hematological parameters i.e., $\mathrm{Hb}, \mathrm{MCV}, \mathrm{MCH}$, Serum Iron, TIBC, RDW and peripheral smear was significantly associated with cases (43\%) when compared with controls $(10 \%)$ (P value 0.0001).Respiratory tract infections were the most common underlying illness among the seizure group, followed by viral fevers, acute diarroheal diseases and urinary tract infections, in the study.Family history of febrile seizures was a significant non modifiable risk factor in the seizure group. $21.4 \%$ of seizure group had a family history of febrile seizures in first degree relatives. There was a significant difference in the value of $\mathrm{Hb}, \mathrm{MCH}$, serum iron, TIBC and RDW among the seizure group as compared to control group. There was a mean difference of $5 \%$ of MCV between the seizure group and controls, which was not statistically significant in the study. Iron deficiency anemia as defined by hematological parameters i.e., $\mathrm{Hb}, \mathrm{MCV}$, $\mathrm{MCH}$, serum iron, TIBC, Retiulocyte count, RDW and peripheral smear with simple febrile seizures in the age group 6-60 months. Complete Blood count, being a simple and feasible investigation, can be used to screen individuals with iron deficiency in simple febrile seizures, for making a decision to start iron supplementation. Our study concludes that $80 \%$ of the study population within seizure group was between 6 months and 36 months. There was no significant gender predisposition for febrile seizures, and both genders were affected more or less in equal proportion. Respiratory tract infections were the most common underlying illness among the seizure group, followed by viral fevers, acute diarroheal diseases and urinary tract infections, in the study. Family history of febrile seizures was a significant non modifiable risk factor in the seizure group. $21.4 \%$ of seizure group had a family history of febrile seizures in first degree relatives. There was a significant difference in the value of $\mathrm{Hb}, \mathrm{MCH}$, serum iron, TIBC and RDW among the seizure group as compared to control group. There was a mean difference of $5 \%$ of $\mathrm{MCV}$ between the seizure group and controls, which was not statistically significant in the study. Iron deficiency anemia as defined by hematological parameters i.e., $\mathrm{Hb}, \mathrm{MCV}, \mathrm{MCH}$, serum iron, TIBC, Retiulocyte count, RDW and peripheral smear with simple febrile seizures in the age group 6-60 months. Complete Blood count, being a simple and feasible investigation, can be used to screen individuals with iron deficiency in simple febrile seizures, for making a decision to start iron supplementation. Role of correction of Iron deficiency in preventing simple febrile seizures in children needs to be studied.

Our study concludes that $80 \%$ of the study population within seizure group was between 6 months and 36 months. There was no significant gender predisposition for febrile seizures, and both genders were affected more or less in equal proportion. Respiratory tract infections were the most common underlying illness among the seizure group, followed by viral fevers, acute diarroheal diseases and urinary tract infections, in the study. Family history of

Volume 6 Issue 1, January 2017 


\section{International Journal of Science and Research (IJSR) \\ ISSN (Online): 2319-7064}

Index Copernicus Value (2015): 78.96 | Impact Factor (2015): 6.391

febrile seizures was a significant non modifiable risk factor in the seizure group. $21.4 \%$ of seizure group had a family history of febrile seizures in first degree relatives. There was a significant difference in the value of $\mathrm{Hb}, \mathrm{MCH}$, serum iron, TIBC and RDW among the seizure group as compared to control group. There was a mean difference of $5 \%$ of MCV between the seizure group and controls, which was not statistically significant in the study. Iron deficiency anemia as defined by hematological parameters i.e., $\mathrm{Hb}, \mathrm{MCV}$, $\mathrm{MCH}$, serum iron, TIBC, Retiulocyte count, RDW and peripheral smear with simple febrile seizures in the age group 6-60 months. Complete Blood count, being a simple and feasible investigation, can be used to screen individuals with iron deficiency in simple febrile seizures, for making a decision to start iron supplementation. Role of correction of Iron deficiency in preventing simple febrile seizures in children needs to be studied.

This study has some limitations. A case-control study of this nature can introduce selection bias because all cases of febrile seizures may not be coming to the hospital. So study be misleading if cases were identified from hospital admission to hospital was influenced not only by the presence and severity of disease but also by other variables, such as social class. Iron status of the body was not determined, as the study was planned to identify iron deficiency, as defined by the hematological parameters. So the specificity of diagnosing iron deficiency was less because other cases of microcytic hypochromic anaemic like Beta thalassemia and lead poisoning could not be ruled out in these cases. Accordingly, there is a need for further large cohort study for this problem.

The strength of this study included standardized criteria for diagnosing febrile seizures, and iron deficiency anemia defined by hematological parameters, concurrent enrollment of cases and controls, and no recall bias regarding exposure. Parameters such as ferritin, transferring saturation and free erythrocyte protoporphyrin were not taken into consideration. These measurements of iron status are influenced by infections and therefore are not reliable indicators of iron status in the settings in the settings of acute infections. (18) Hematological parameters have been demonstrated to be sensitive and specific in children, and stable in the presence of febrile illness, (19) and are therefore strength of the study design.

Furthermore, whether correction of iron deficiency reduces the incidence of febrile seizures is an attractive hypothesis that needs to be tested.

\section{References}

[1] Johnston MV. Seizures in childhood. In: Kleigman RM. Behrman RE, Jenson HB, Station BP, Nelson Text Book of Pediatrics $18^{\text {th }}$ Edition Philadeplhia: Saunders Elsevier : 2007.p.2457-8.

[2] Camfield P, Camfield C, Gordon K.Antecedents and risk factors for febrile seizures. San Diego, CA:Academic press;2002.

[3] Rich SS, Annegers JF, Hauser WA, Anderson VE. Complex segregation analysis of febrile convulsion. Am J Hum Genet 1987;41:249-57.
[4] Tsuboi T.Genetic analysis of febrile convulsions: twin and family studies. Hum Genet 1987;75:7-14.

[5] Johnson WG, Kugler SL, Stenroos ES, Meulener MC, Rangwalla I, Johnson TW, et al. Pedigree analysis in families with febrile seizures. Am J Med Genet 1996;61:345-52.

[6] Angulo- Kinzler RM, Peirano P, Lin E, Algarin C, Garrido M, Lozoff B. Twentyfour-hour motor activity in human infants with and without iron deficiency anemia. Early Hum Dev 2002; 70(1-2) :85-101.

[7] DeMaeyer E, Adiels-Tegman M. The prevalence of anemia in the world. World Health Stat Q 1985; 38:302-16.

[8] Florention RF, Guirriee RM, Stekel A. Prevalence of nutritional anemia in infancy and childhood with emphasis on developing countries. Iron nutrition in infancy and childhood. New York: Raven Press; 1984.p.61-74.

[9] Freire W. Strategies of the Pan American Health Organization- World Health Organization for the control of iron deficiency in Latin America. Nutr Rev 1997; 55:183-8.

[10] World Health Organization. Iron Deficiency Anemia. Assessment, Prevention and Control. A guide for program Managers. WHO/NHD/013; Geneva:2001.

[11] American Academy of Pediatrics, Steering Committee on Quality Improvement and Management. Classifying recommendations for clinical practice guidelines. Pediatrics. 2004; 114:874-7...

[12] Dawn SH, Jonatan T, Jerome Y, Don S, Haines C, Craig WR. The association between iron deficiency and febrile seizure in childhood. Clin Pediatr. 2009; 48: 420-6

[13] Daoud AS, Batieha A, Abu-Ekteish F, Gharaibeh N, Ajlouni S, Hijazi S. Iron status: a possible risk factor for the first febrile seizure. Epilepsia 2002; 43(7) : 740-3.

[14] Kobrinsky NL, Yager JY, Cheang MS, Yatscoff RW, Tenenbein M.Does iron deficiency raise the seizure threshold. J child Neurol 1995; 10(2) : 105-9.

[15] Bidabadi E, Mashouf M. Association between iron deficiency anemia and first febrile convulsion: A casecontrol study seizure 2009; 18: 347-51.

[16] H Derakhshanfer - 2012 ,Medcglas (Zenica).2012 august 9 (2): 239-42-Association between iron deficiency anemia febrile seizure in children.16

[17] Dawn.s. Hartfield Jonathan Tan,Jerome y yager,Rhonda J. Rasychuk,Don spady,Christa hames and Willian R. craig-The Association between Iron deficiency febrile seizures in childhood.17

[18] Kin SK, Cheong WS, Jun YH, Red blood cell indices and Iron status according to feeding practices in infants and young children. Acta Paediatrics. 1996;85;139$144 . .18$

[19] Recommendations to prevent and control iron deficiency in the United States. MMWR Morb Mortal Wkly Rep.1998;47:1-36.19 